\title{
Hybrid-PIC Simulation on Plasma Flow of Hollow Cathode
}

\author{
By Kenichi Kubota, ${ }^{1)}$ Yuya Oshio, ${ }^{2)}$ Hiroki Watanabe, ${ }^{3)}$ Shinatora Cho, ${ }^{1)}$ Yasushi OhkawA,,${ }^{4)}$ and Ikkoh Funaki ${ }^{2)}$ \\ ${ }^{1)}$ Aeronautical Technology Directorate, JAXA, Chofu, Tokyo, Japan \\ ${ }^{2}$ Institute of Space and Astronautical Science, JAXA, Sagamihara, Kanagawa, Japan \\ 3) Faculty of System Design, Tokyo Metropolitan University, Hino, Tokyo, Japan \\ ${ }^{4)}$ Research and Development Directorate, JAXA, Chofu, Tokyo, Japan
}

(Received July 31st, 2015)

\begin{abstract}
In order to understand plasma properties of hollow cathodes, a numerical simulation code with Hybrid-PIC model has been developed, in which ions and electrons are modeled as particles and fluid, respectively. In this study, as a first step, the applicability of the model is demonstrated, and then the influences of the emitter temperature on the flow field are discussed for a discharge current of $30 \mathrm{~A}$ and a mass flow rate of $1 \mathrm{mg} / \mathrm{s}$. The electron density for the maximum emitter temperature of $1900 \mathrm{~K}$ agrees well with the experimental data from JPL. The results also show that the electron density tends to be higher with lower emitter temperature due to the higher electron temperature inside the cathode tube. The higher electron temperature is caused by the energy loss suppression resulting from the higher sheath voltage on the emitter surface. It was also found that charge exchange collisions shift the location of the electron density peak upstream.
\end{abstract}

Key Words: Electric Propulsion, Hollow Cathode, Simulation, Hybrid-PIC

\section{Nomenclature}

$\begin{array}{ccl}A & : & \text { Richardson-Dushman Coefficient } \\ e & : & \text { elementary charge } \\ \boldsymbol{E} & : & \text { electric field } \\ j & : & \text { current density } \\ k & : & \text { Boltzmann constant } \\ L & : & \text { characteristic length } \\ n & : & \text { number density } \\ m & : & \text { mass of particle } \\ p & : & \text { pressure } \\ T & : & \text { temperature } \\ \boldsymbol{u} & : & \text { velocity } \\ \mathcal{E}_{0} & : & \text { permittivity of vacuum } \\ \mu & : & \text { mobility } \\ v & : & \text { collision frequency } \\ \phi & : & \text { electric potential } \\ \text { Subscripts } & \\ \mathrm{e} & : & \text { electron } \\ \text { em } & : \text { emitter } \\ \text { i } & : \text { ion } \\ \text { IAT } & : \text { ion acoustic turbulence } \\ \mathrm{n} & : \text { neutral } \\ \text { sh } & : \text { sheath } \\ \text { W } & : \text { work function } \\ & & \end{array}$

\section{Introduction}

Recent years, research and development of high power Hall thrusters have been activated to apply them to all-electric satellites and/or deep-space explorers. To shorten trip time, a thrust of several hundred $\mathrm{mN}$ should be attained, which leads to requirement of a cathode which can provide electron current about several tens of amperes or more.

A number of experimental, theoretical and numerical efforts on hollow cathodes for electric propulsion have been made for years by researchers, ${ }^{1-17)}$ and overall characteristics of hollow cathode plasma are being unveiled. One of the most serious issues on the lifetime of cathodes is sputtering of a keeper electrode by energized ions. ${ }^{2,3,18-22)}$ Most probable origins of the high-energy ions is the ion acoustic turbulence (IAT), first predicted to exist and to generate anomalous resistivity and high energy ions in cathodes by OrCa2D simulations ${ }^{23-25)}$ which utilizes fluid model coupled with collisionless neutral model in the plume region. ${ }^{26)}$ The propagation of the IAT and its damping which causes the high-energy ions is related with the kinetics of heavy particles (neutrals and ions). ${ }^{20-22)}$ Another candidate of the origins of the energized ions is a potential hill formed downstream of the cathode orifice as a result of extensive electron-impact ionization, which accelerates ions created in the hill spherically through the potential fall that develops around the hill crest. ${ }^{2,3,13)}$ In either case, kinetics of heavy particles should be pursued at least in order to capture the phenomenon which averts the ion distribution function from Maxwellian.

Developing a hollow cathode, we believe numerical simulations as well as experiments are quite beneficial to understand substantial physics in cathodes and to optimize working conditions. To take into account the heavy particle's kinetics in a natural way, we are developing a numerical code with a Hybrid-PIC (Particle In Cell) model, in which heavy particles and electrons are modeled as particles and fluid, respectively. The objectives of this study are to demonstrate the applicability of Hybrid-PIC model for the hollow cathode simulation as a first step, whereas the detailed discussion on the energized ions is not covered at the present stage. The 
flow properties and influences of the emitter temperature on the flow field are discussed under the condition of a constant discharge current and a constant mass flow rate, because the emitter temperature has an uncertainty depending on the thermal insulation property around the emitter itself even for a fixed working condition. The electron density profiles are compared to the experimental data to validate our results. The role of CEX collisions in the electron density profile is also discussed.

\section{Numerical Modeling}

\subsection{Hybrid-PIC model}

The flow was assumed to be axisymmetric and quasi-neutral in the bulk region. The kinetics of ions was traced with PIC method and collisions were dealt with DSMC (Direct Simulation Monte Carlo) method, where charge exchange collision (CEX) and elastic collisions (n-n, n-i, i-i) were taken into account. The viscosity in the neutrals which has significant impact on the flow ${ }^{27)}$ is naturally implemented via DSMC model. Ionization process takes place by electron impact on neutrals, where multiply charged ions were ignored. Since the neutral density is quite higher than the ion density, a typical weight of a macroneutral was set much larger than that of a macroion. Thus the mass decay model for neutrals was used to implement the ionization process. ${ }^{28)} \mathrm{CEX}$ is often modeled as "identity switch" between a neutral and an ion without further interaction, but such a model neglects interaction via the polarization potential which can lead to a large scattering angle. In this study, CEX and elastic collision were implemented with the united theory proposed by Nanbu, ${ }^{29)}$ in which the interaction through the polarization potential is simply modeled in a stochastic way. The model includes two free parameters: cut-off dimensionless impact parameter and a coefficient to determine a maximum impact parameter effective for CEX. In this study, the former was assumed to be 9 as proposed in the original literature. As to the latter parameter, Nanbu determined it for $\mathrm{He}, \mathrm{Ne}, \mathrm{Ar}$, and $\mathrm{Kr}$ so that the drift velocity resulting from the model agrees with the experimental data. The resultant values are 2.6 for $\mathrm{He}$, $\mathrm{Ne}$, Ar, and 2.5 for $\mathrm{Kr}$. Since the value for $\mathrm{Xe}$ is not available, we assumed the same value as $\mathrm{Kr}$.

The electrons were modeled by the drift-diffusion model.

$$
\boldsymbol{u}_{e}=-\mu_{e} \boldsymbol{E}-\frac{\mu_{e}}{e} \frac{\nabla p_{e}}{n_{e}}, \quad \boldsymbol{E}=-\nabla \phi
$$

The electron number density is equal to the ion number density determined by ion PIC due to the quasi-neutral assumption. In the calculation of the electron mobility, the effect of anomalous resistivity due to IAT was included, ${ }^{7)}$

$$
\mu_{e}=\frac{e}{m_{e} v_{e}}, \quad v_{e}=v_{e n}+v_{e i}+v_{I A T}, \quad v_{I A T}=\alpha \omega_{p, i} \frac{\left|\boldsymbol{u}_{e}\right|}{c_{i}}
$$

where $\omega_{p, i}$ and $c_{i}$ are ion plasma frequency and ion acoustic velocity, respectively. The factor of $T_{e} / T_{i}$ appeared in the original formula of $v_{I A T}$ was assumed to be included in the coefficient $\alpha$ in accordance with 7). In all results shown here, $\alpha$ was empirically set at 0.001 . Since Eq. (1) is derived from the electron's kinetic equation, ${ }^{30)}$ the micro-instability of electrons resulting in generation of IAT is implicitly incorporated as the resistivity model which affects the electric potential and subsequent ion kinetics. By substituting Eq. (1) into the current conservation law, we obtain the elliptic differential equation to determine the electric potential.

$$
\nabla \cdot e n_{e}\left(\boldsymbol{u}_{i}-\boldsymbol{u}_{e}\right)=0 \rightarrow \nabla \cdot n_{e} \mu_{e} \nabla \phi=\nabla \cdot\left(n_{e} \boldsymbol{u}_{i}+\frac{\mu_{e}}{e} \nabla p_{e}\right) \text { (3) }
$$

The electron temperature is given from the steady-state energy conservation equation,

$$
\nabla \cdot\left(\frac{5}{2} n_{e} k T_{e} \boldsymbol{u}_{e}+\boldsymbol{q}_{e}\right)=\boldsymbol{j}_{e} \cdot \boldsymbol{E}-Q_{i o n}-Q_{e x c}
$$

where $Q_{i o n}$ and $Q_{e x c}$ represent energy consumption due to ionization and excitation, respectively. ${ }^{31)}$ Eqs. (3) and (4) were discretized with finite element methods and were solved by means of a direct solver to enhance numerical stability. As a system solver, we utilized the parallelized sparse direct solver of MUMPS. ${ }^{32)}$ The solver to trace particle dynamics is also parallelized by means of domain decomposition, where the computational domain is dynamically decomposed to make a load factor of each process uniform.

\subsection{Computation target}

Since we have not obtained our own experimental data, we assumed imaginary cathode as the simulation target. The inner diameter and the axial length of the emitter were $6.3 \mathrm{~mm}$ and $25 \mathrm{~mm}$, respectively, which are the same values as those of $1.5 \mathrm{~cm}$-class cathode of JPL. ${ }^{33,34)}$ Since its plasma parameters had been closely investigated in the wide operating range, we can utilize them to validate the simulation results. The cathode and keeper orifice diameters were $4 \mathrm{~mm}$ and $6.3 \mathrm{~mm}$ in this simulation. To reduce the computational cost, an imaginary flat anode was assumed in the plume region, whereas a cylindrical anode was used in the JPL's experiment.

\subsection{Boundary conditions}

At the inlet boundary, the working gas is fed uniformly as neutrals and has an assumed temperature of $500 \mathrm{~K}$. Once an ion collides with the wall, it is recombined with an electron and reflected as a neutral. Then some macroions are merged so as to create one macroneutral due to the weight disparity. We can freely set an accommodation factor to specify the degree of thermalization to the wall temperature, but the results shown here assumed an accommodation factor as unity, i.e. the particles are completely thermalized to the wall temperature. On the wall boundaries, the ion/electron density is modified so as to satisfy the Bohm condition. On the emitter surface, the temperature profile was assumed to be the measured one obtained by JPL. ${ }^{7}$ With the fitted curve of the normalized profile shown in Fig. 1, the maximum temperature is parametrically varied as mentioned later. On the plume boundaries, while the neutrals penetrate the boundary, the ions are reflected. This is because, according to the plasma diagnosis, the further apart from the cathode orifice, the higher the electric potential become. ${ }^{35)}$ When the ion density becomes 
lower than a lower limit value on the plume boundary, macroions are supplemented so as to maintain the limit value.

The assumption of quasi-neutrality requires sheath models on the wall boundaries. In this simulation, the sheath edge, which means the boundary between the sheath and the pre-sheath region as shown in Fig. 2, corresponds to the computational boundary. One of the most important boundaries is the emitter surface on which thermionic emission should be included into the sheath model. On this boundary, the following electron flux was assumed.

$$
n_{e} \boldsymbol{u}_{e} \cdot \boldsymbol{n}=\frac{1}{4} n_{e} \sqrt{\frac{8 k T_{e}}{\pi m_{e}}} \exp \left(-\frac{e \phi_{s h}}{k T_{e}}\right)-\frac{j_{e m}}{e}
$$

The first term in the right hand side is the electron loss from the bulk plasma, and the second term represents the contribution of the thermionic electrons which can be evaluated with Richardson-Dushman formula including Schottky effect,

$$
j_{\text {em }}=A T_{e m}^{2} \exp \left(\frac{-e \phi_{\text {eff }}}{k T_{\text {em }}}\right), \quad \phi_{\text {eff }}=\phi_{w}-\sqrt{\frac{e\left|E_{\text {em }}\right|}{4 \pi \varepsilon_{0}}}
$$

where $A, T_{e m}$ and $E_{e m}$ represent Richardson-Dushman coefficient, emitter temperature and electric field on the emitter surface, respectively. Note that $j_{e m}$ is an absolute value of the emission current. The formula to evaluate $E_{\text {em }}$ can be obtained by integrating Poisson equation (Gauss's law). Since $E_{e m}$ depends on $j_{e m}$, Eq. (6) should be iteratively solved with regard to $j_{e m}$. The $E_{e m}$ also depends on the ion velocity at the sheath edge, where the ions were assumed to have Bohm velocity corrected due to the effect of thermionic emission. ${ }^{36}$ ) Equation (5) is substituted into the electron flux of Eq. (3) on the emitter boundary. On the cathode orifice boundary, we only have to vanish the second term of the right hand side of Eq. (5). The keeper electrode was assumed to float in accordance with the experiment, ${ }^{34)}$ i.e. the keeper voltage was adjusted so as to keep the keeper current zero. On the plume boundary, in order to simulate discharge with a plate anode located downstream, the electric potential was set to a discharge voltage $V_{d}$ at $x / L_{e m}=2.8$, whereas $V_{d}$ was periodically adjusted so that the discharge current can be kept around a specified value. On the other hand, no current condition was imposed on the upper boundary at $y / L_{e m}=0.8$.

The electron thermal flux at the sheath edge of the emitter surface can be evaluated as follows.

$$
\begin{aligned}
q_{e}= & \frac{1}{4} n_{e} \sqrt{\frac{8 k T_{e}}{\pi m_{e}}} \exp \left(-\frac{e \phi_{s h}}{k T_{e}}\right)\left(2 k T_{e}+e \phi_{s h}\right) \\
& -\frac{j_{e m}}{e}\left(2 k T_{e m}+e \phi_{s h}\right)
\end{aligned}
$$

On the other wall boundaries, we can vanish the second term of the right hand side. On the inlet and plume boundaries, the electron temperature was fixed at assumed values.

\subsection{Calculation conditions}

The calculation conditions are tabulated in Table 1. The working gas was Xe fed at a mass flow rate of $1 \mathrm{mg} / \mathrm{s}$. The $\mathrm{LaB}_{6}$ was selected as emitter material ${ }^{33,37)}$ and its maximum temperature $T_{e m, \max }$ was parametrically varied between 1850 and $1950 \mathrm{~K}$ for a constant discharge current of $30 \mathrm{~A}$ in order to understand qualitative influences of the emitter temperature on the flow field. The keeper electrode was floated in accordance with the experiment condition at JPL. ${ }^{33)}$ The inlet and outlet (plume boundary) electron temperatures, and the lower limit of the electron number density in the plume region were fixed at $1,3 \mathrm{eV}$, and $5 \times 10^{17} \mathrm{~m}^{-3}$, respectively. The influence of these parameters on the results should be surveyed in the future.

Figure 3 shows the computational domain used in this simulation. In the Hybrid-PIC model, the mesh size should be equal to or less than the mean free path of the particle collisions. The minimum mesh size in the emitter region used in the present study was set at $0.1 \mathrm{~mm}$. This is much less than mean free paths with regard to the neutral-ion collisions including CEX.

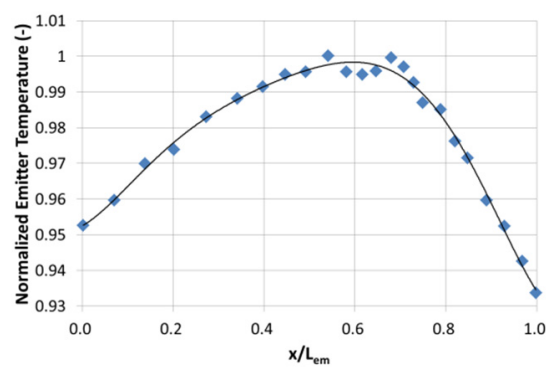

Fig. 1. Normalized emitter temperature profile.?

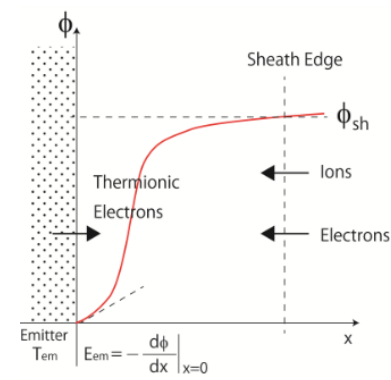

Fig. 2. Sheath with thermionic emission on emitter surface.

Table 1. Calculation Conditions.

\begin{tabular}{|l|l|}
\hline Gas & Xenon \\
\hline Emitter material (work function) & $\mathrm{LaB}_{6}(2.67 \mathrm{eV})$ \\
\hline Mass flow rate & $1 \mathrm{mg} / \mathrm{s}$ \\
\hline Discharge current & $30 \mathrm{~A}$ \\
\hline Maximum emitter temperature & $1850-1950 \mathrm{~K}$ \\
\hline Keeper condition & Floated \\
\hline Inlet electron temperature & $1 \mathrm{eV}$ \\
\hline Outlet electron temperature & $3 \mathrm{eV}$ \\
\hline Lower limit of electron number density & $5 \times 10^{17} \mathrm{~m}^{-3}$ \\
\hline
\end{tabular}

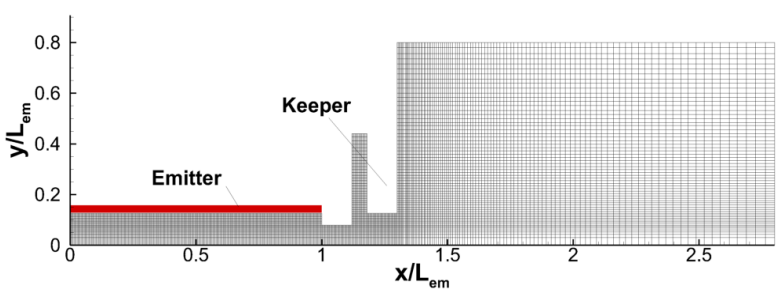

Fig. 3. Computational mesh. 


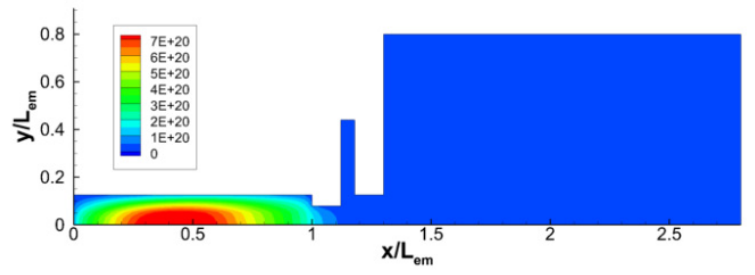

(a) Electron number density, $\mathrm{m}^{-3}$

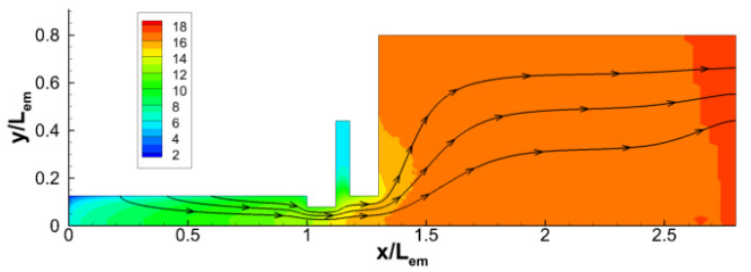

(b) Electric potential with electron streamlines, $\mathrm{V}$

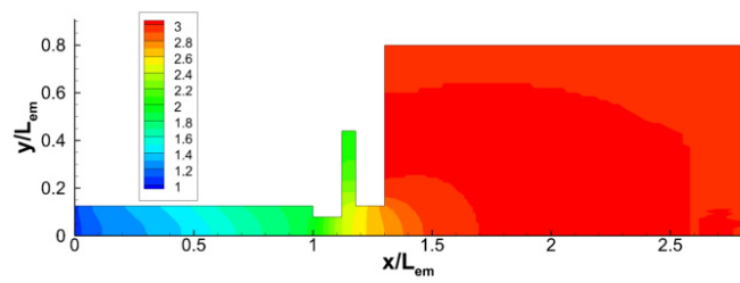

(c) Electron temperature, $\mathrm{eV}$

Fig. 4. Flow field distributions for $T_{e m, \max }=1900 \mathrm{~K}$.

\section{Simulation Results}

\subsection{Discharge field}

Figure 4 shows the electron number density, electric potential, and electron temperature distributions for $T_{e m, \max }=1900 \mathrm{~K}$. The density has a peak about $7 \times 10^{20} \mathrm{~m}^{-3}$ on the centerline around the middle of the emitter region, and declines in the radial and axial direction. In the emitter region, formation of the pre-sheath can be seen, which causes the radial plasma loss on the emitter surface. The electric potential is increased from about 6 to 12 in the emitter region, and then its gradient becomes higher around the orifice region. In the plume region, the density is nearly uniform at the specified limit value. The electric potential grows toward downstream region quite gradually and amounts to about $17 \mathrm{~V}$ in this case. The electron temperature behaves similar to the electric potential, where the temperature ranges from $1 \mathrm{eV}$ to $2 \mathrm{eV}$ in the emitter region, and rapidly increases to about $3 \mathrm{eV}$ through the orifice region, which corresponds to the specified value.

\subsection{Influence of emitter temperature}

Figure 5 shows the electron density, electric potential, and electron temperature profiles along the centerline as a function of the maximum emitter temperature $T_{\text {em,max }}$. We can see that the electron number density inside the cathode tube tends to be lower with higher $T_{e m, \max }$. While the top of the profile moves toward downstream direction as $T_{e m, \max }$ increases, the profiles overlap with each other in the range of $x / L_{e m}>1$, and the density approaches to the specified lower limit value in the end. Figure 6 shows comparison of the electron density inside the cathode tube between the simulation results and the experiment by JPL. ${ }^{34)}$ This indicates that the simulation result

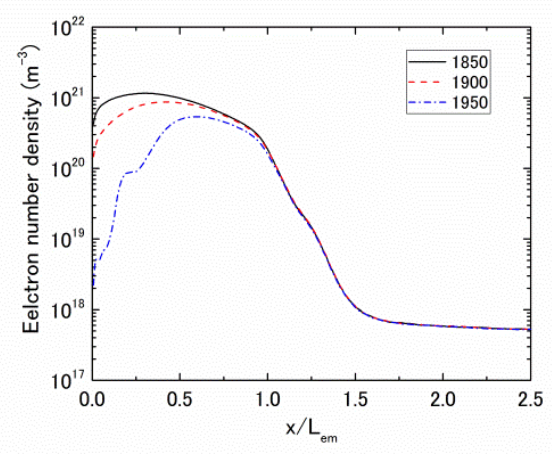

(a) Electron number density, $\mathrm{m}^{-3}$

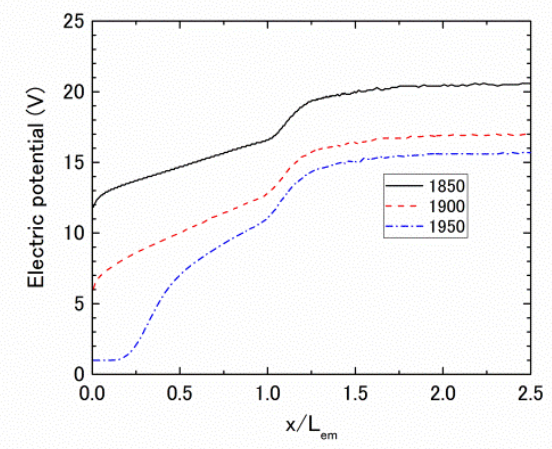

(b) Electric potential with electron streamlines, $\mathrm{V}$

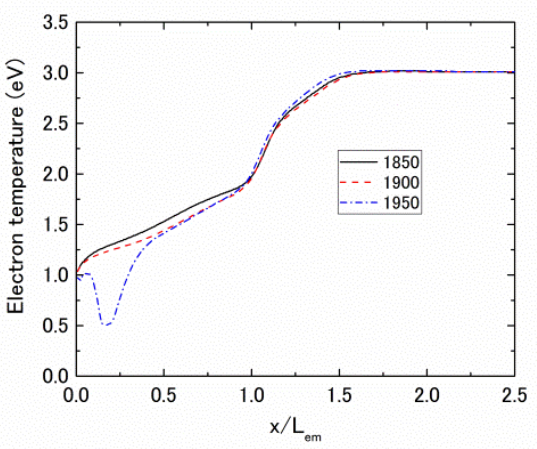

(c) Electron temperature, $\mathrm{eV}$

Fig. 5. Plasma characteristics along centerline for $T_{e m, \max }=1850,1900$, $1950 \mathrm{~K}$.

for $T_{e m, \max }=1900 \mathrm{~K}$ has most similar profile to the measured one in terms of not only qualitative but also quantitative aspect. The emitter temperature of $1950 \mathrm{~K}$ seems unrealistic for the present conditions.

The electric potential profile inside and outside the cathode tube is significantly affected by the $T_{e m, \max }$. On the whole, the potential has positive gradient and the gradient becomes steep locally around the orifice region. The asymptotic values of the potential for $T_{e m, \max }=1850$ and $1900 \mathrm{~K}$ at the plume region are 20.5 and $17.0 \mathrm{~V}$, respectively. Since the computational domain in the plume region is limited and we have no information about the anode sheath, fair comparison of the discharge voltage with the experiment is impossible, but these obtained values are not far from the measured value about 21 $\mathrm{V}$ for 30 A. $^{34)}$

The electron temperature profiles for $T_{e m, \max }=1850$ and $1900 \mathrm{~K}$ are nearly the same as a whole, whereas the line for 


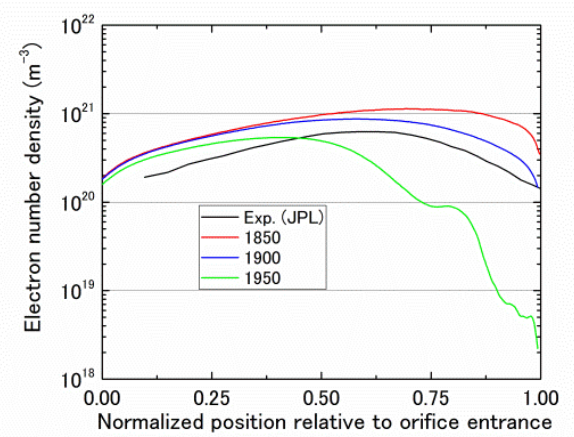

Fig. 6. Comparison of electron number density inside the cathode tube for $T_{e m, \max }=1850,1900,1950 \mathrm{~K}$ between simulation and experiment by JPL. ${ }^{34)}$

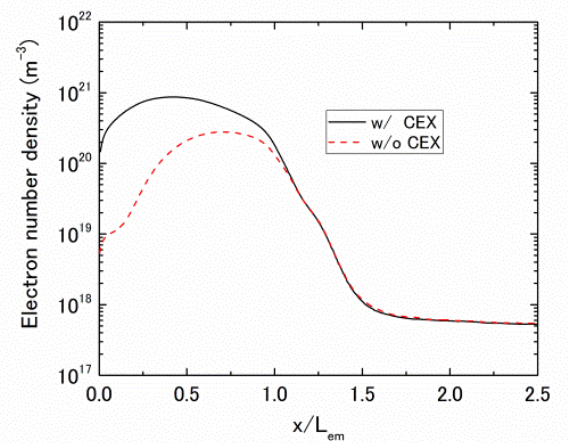

Fig. 7. Electron number density along centerline with and without CEX model for $T_{e m, \max }=1900 \mathrm{~K}$.

$1850 \mathrm{~K}$ is slightly higher in the cathode tube. The profile for $1950 \mathrm{~K}$ has a undershoot in the cathode tube. This may be due to the excess energy loss on the emitter wall caused by the low sheath potential, and the inappropriate electron temperature fixed at the inlet.

\section{Discussion}

\subsection{Electron density distribution}

In Fig. 4-(a), the electron density has a peak around $x / L_{e m}=$ 0.4 , which is consistent with the measured profile as seen from Fig. 6. The location of the density peak is affected by CEX collision. To clarify the role of the CEX collision in the flow field, the electron density profiles with and without CEX collision are compared in Fig. 7. We can see that, if CEX is ignored, the peak location shifts downstream, which is attributed to the heavy particle's behavior. Figure 8 shows the streamlines of neutrals and ions inside the cathode tube with and without CEX. Without CEX, the neutrals have basically positive velocity in $\mathrm{x}$ direction. The streamlines extended from the emitter surface is due to the neutrals produced by recombination, i.e. ions are attracted to the emitter surface by the potential gradient in the pre-sheath, and turn into neutrals via recombination with electrons. On the other hand, when CEX is considered, the streamlines of neutrals are folded back, which generates the vortex near the inlet. This is because the neutrals initially flowing downstream are supposed to be

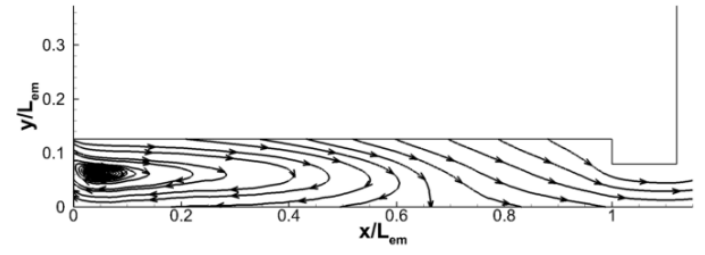

(a) Neutral with CEX

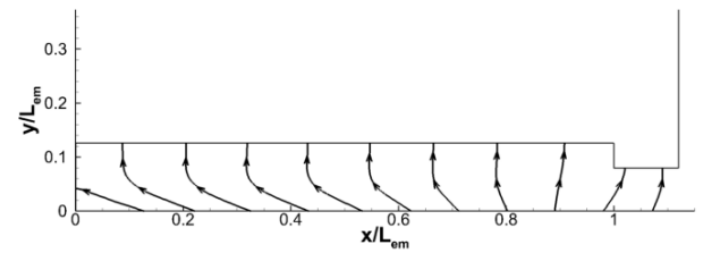

(b) Ion with CEX

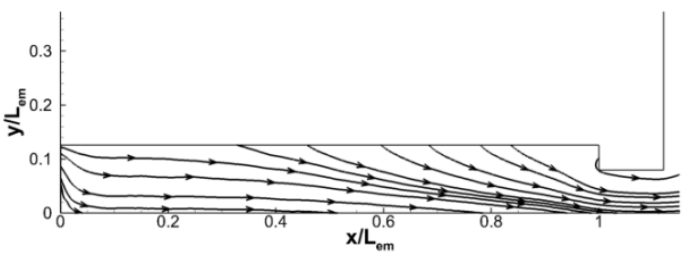

(c) Neutral without CEX

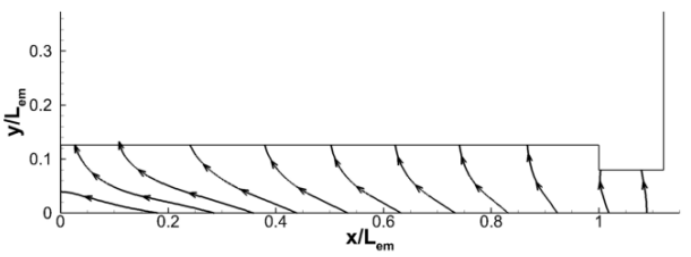

(d) Ion without CEX

Fig. 8. Streamlines of neutrals and ions with and without CEX model for Tem, $\max =1900 \mathrm{~K}$.

replaced with ions flowing upstream by CEX collisions, then the new neutrals tend to be directed in $-\mathrm{x}$ direction especially near the centerline where the ion density is relatively high. This mechanism plays a role in rising neutral density near the inlet, which contributes to increasing ion/electron density and consequently to the shift of the density peak location.

\subsection{Influences of emitter temperature}

To clarify why the electron number density inside the cathode tube tends to be lower with higher $T_{e m, \max }$, loss of the electrons is discussed in terms of the electric potential. The electric potential inside the cathode tube is basically sustained by the electron-repelling sheath formed on the emitter surface. The influence of the emitter temperature on the sheath voltage can be qualitatively understood from the equation of current conservation at the sheath edge.

$$
\begin{aligned}
& j_{t o t}=j_{i}-j_{e}+j_{e m}=j_{i}-j_{t h} \exp \left(-\frac{e \phi_{s h}}{k T_{e}}\right)+j_{e m} \\
& \rightarrow \phi_{s h}=-\frac{k T_{e}}{e} \ln \left(\frac{j_{i}-j_{t o t}+j_{e m}}{j_{t h}}\right)
\end{aligned}
$$

where

$$
j_{t h}=\frac{1}{4} e n_{e} \sqrt{\frac{8 k T_{e}}{\pi m_{e}}}
$$


Here $j_{t o t}, j_{i}, j_{e}$, and $j_{e m}$ are a total current, ion current, plasma electron current, and emission current, respectively. Note that each current denotes an absolute value. If the ion velocity at the sheath edge toward the wall is assumed to be the standard Bohm velocity $\left(=\sqrt{k T_{e} / m_{i}}\right)$, and the Schottky effect is ignored, the sheath voltage can be calculated from Eq. (8) as a function of the emitter temperature for constant $j_{t o t}, n_{e}$ and $T_{e}$. When $n_{e}=10^{20} \mathrm{~m}^{-3}$ and $T_{e}=2 \mathrm{eV}$, the relation between the sheath voltage and the emitter temperature for $j_{\text {tot }}=10^{4}$ and $10^{5} \mathrm{~A} / \mathrm{m}^{2}$ can be depicted like in Fig. 9. Depending on $j_{t o t}$, the $\phi_{s h}$ takes on quite different characteristics. This can be understood from Eq. (8), i.e. when $j_{t o t}<j_{i}$, the $\phi_{s h}$ can be kept finite even at a low emitter temperature, but otherwise, the $\phi_{s h}$ is to diverge at a certain cathode temperature. From these curves, we can presume the $\phi_{s h}$ tends to be higher with lower emitter temperature regardless of $j_{\text {tot }}$ around $T_{\text {em, } \max }=1900 \mathrm{~K}$. This is why the electric potential becomes lower with higher emitter temperature as seen in Fig. 5(b).

The lower sheath potential on the emitter will accept more electrons, which leads to increase in the electron's thermal loss into the wall surface. The electron heat loss estimated from the first term of Eq. (7) was increased from $20 \mathrm{~W}$ for $T_{e m, \max }=1850 \mathrm{~K}$ to $104 \mathrm{~W}$ for $T_{e m, \max }=1900 \mathrm{~K}$. This causes the lower electron temperature inside the cathode tube for higher emitter temperature as seen in Fig. 5(c). The lower electron temperature discourages ionization processes, thus the electron density for higher emitter temperature becomes lower.

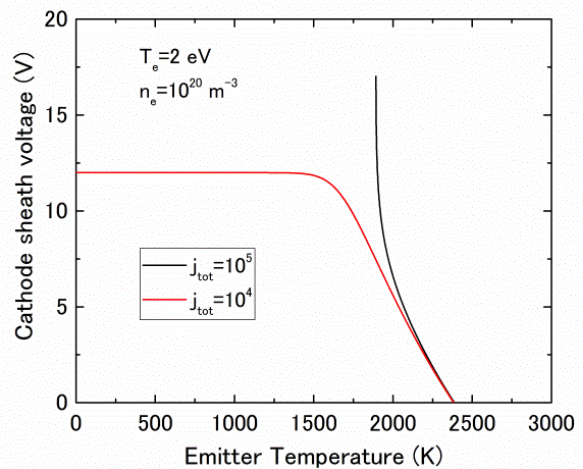

Fig. 9. Relation between cathode sheath voltage and cathode temperature: The ion velocity is assumed to be standard Bohm velocity, and Schottky effect is ignored.

\section{Conclusion}

The plasma flow of the hollow cathode was simulated with the newly developed Hybrid-PIC code which models ions and electrons as particles and fluid, respectively. In this study, the applicability of the model for the hollow cathode was demonstrated, and the influences of the emitter temperature on the flow field were discussed under a constant discharge current of $30 \mathrm{~A}$ and a mass flow rate of $1 \mathrm{mg} / \mathrm{s}$ as a first step. The emitter temperature $T_{e m, \max }$ was parametrically varied from 1850 to $1950 \mathrm{~K}$. The inner diameter and the length of the emitter were set to the same as those of $1.5 \mathrm{~cm}$ class cathode of JPL, which enables us to compare our results to the available experimental data.
The comparison with the experimental data shows that the electron density profile in the emitter region for $T_{e m, \max }=$ $1900 \mathrm{~K}$ agrees well with the measured data, which suggests the applicability of the model to the hollow cathode plasma. As the emitter temperature decreases, the electric potential becomes higher, and then the electron energy loss through the electron repelling sheath is suppressed, which leads to the higher electron temperature, and hence higher electron number density. It was also found that CEX collision makes the neutrals pushed back toward upstream in the middle of the cathode tube, which strongly affects the electron density profile.

\section{Acknowledgments}

This research has been supported by a Grant-in-Aid for Young Scientists (B) (26820377) from the Japan Society for Promotion of Science.

\section{References}

1) Brophy, J R., and Garner, C. E.: Tests of High Current Hollow Cathodes for Ion Engines, AIAA Paper 88-2913, 1988.

2) Friendly, V. J., and Wilbur, P. J.: High Current Hollow Cathode Phenomena, Journal of Propulsion and Power, 8(1992), pp. 635-643.

3) Kameyama, I.: Effects of Neutral Density on Energetic Ions Produced Near High-current Hollow Cathodes, NASA Contractor Report 204154, 1997.

4) Mandell, M. J., and Katz, I.: Theory of Hollow Cathode Operation in Spot and Plume Modes, AIAA Paper 94-3134, 1994.

5) Katz, I., Mikellides, I. G., and Goebel, D. M.: Model of the Plasma Potential Distribution in the Plume of a Hollow Cathode, AIAA Paper 2004-4108, 2004.

6) Goebel, D. M., and Chu, E.: High-current Lanthanum Hexaboride Hollow Cathode for High-power Hall Thrusters, Journal of Propulsion and Power, 30 (2014), pp. 35-40.

7) Mikellides, I. G., Goebel, D. M., Jorns, B. A., Polk, J. E., and Guerrero, P.: Numerical Simulations of the Partially-ionized Gas in a 100-A LaB6 Hollow Cathode, IEPC Paper 2013-142, 2013.

8) Mikellides, I. G., and Katz, I.: Wear Mechanisms in Electron Sources for Ion Propulsion, I: Neutralizer Hollow Cathode, Journal of Propulsion and Power, 24(2008), pp. 855-865.

9) Coletti, M., and Gabriel, S. B.: Emitter Temperature Measurements of a 180A Hollow Cathode for the HiPER Project, AIAA Paper 2012-4081, 2012.

10) Thomas, R. E., Kamhawi, H., and Williams, G. J.: High Current Hollow Cathode Plasma Plume Measurements, IEPC Paper 2013-076, 2013.

11) Gaétan, S., Garrigues, L., and Boeuf, J. P.: Hollow Cathode Modeling: a First Approach on Scaling Laws, IEPC Paper 2015-14, 2015.

12) Ohkawa, Y., Higuchi, T., Hayakawa, Y, Miyazaki, K., and Nagano, H.: Observation and Analysis of Graphite Hollow Cathode after 45,000-hour Life Test, IEPC Paper 2013-364, 2013.

13) Farnell, C. C., Williams, J. D., and Farnell, C. C: Comparison of Hollow Cathode Discharge Plasma Configurations, Plasma Sources Science and Technology, 20(2011) 025006.

14) Pedrini, D., Albertoni, R., Paganucci, F., and Andrenucci, M.: Development of $\mathrm{a} \mathrm{LaB}_{6}$ Cathode for High-power Hall Thrusters, IEPC Paper 2015-47, 2015.

15) Kovaleski, S. D., Patterson, M. J., Soulas, G. C., and Sarver-Verhey, T. R.: A Review of Testing of Hollow Cathodes for The International Space Station Plasma Contactor, IEPC Paper 01-271, 2001

16) Bianconi, M., Cirri, G., Brun, M. L., Matticari, G., and Wilbur, P.: A Review of the Manufacturing and Characterization Activities at PROEL of a Plasma Bridge Neutralizer for the RIT 10 Ion Thruster, 
AIAA Paper 90-2588, 1990.

17) Jack, T. M., Patterson, S. W., and Fearn, D. G.: The Effect of the Keeper Electrode on Hollow Cathode Characteristics, AIAA Paper 2000-3533, 2000.

18) Goebel, D. M., Jameson, K., Katz, I., and Mikellides I. G.: Energetic Ion Production and Keeper Erosion in Hollow Cathode Discharges, IEPC Paper 2005-266, 2005.

19) Chu, E., Goebel, D. M., and Wirz, R. E.: Reduction of Energetic Ion Production in Hollow Cathodes by External Gas Injection, Journal of Propulsion and Power, 29(2013), pp. 1155-1163.

20) Jorns, B. A., Mikellides, I. G., and Goebel, D. M.: Temporal Fluctuations in a 100-A LaB6 Hollow Cathode, IEPC Paper 2013-385, 2013.

21) Jorns, B. A., Mikellides, I. G., and Goebel, D. M.: Investigation of Energetic Ions in a 100-A Hollow Cathode, AIAA Paper 2014-3826, 2014.

22) Jorns, B. A., Mikellides, I. G., Goebel, D. M., and Ortega, A L.: Mitigation of Energetic Ions and Keeper Erosion in a High-current Hollow Cathode, IEPC Paper 2015-134, 2015.

23) Mikellides, I. G., Katz, I., Goebel, D. M., and Polk, J. E.: Hollow Cathode Theory and Experiment. II. A Two-dimensional Theoretical Model of the Emitter Region, Journal of Applied Physics, 98(2005), 113303.

24) Mikellides, I. G., Katz, I., Goebel, D. M., and Jameson, K. K.: Evidence of Non-classical Plasma Transport in Hollow Cathodes for Electric Propulsion, Journal of Applied Physics, 101(2007), pp. 063301.

25) Mikellides, I. G., Katz, I., Goebel, D. M., Jameson, K. K., and Polk, J. E.: Wear Mechanisms in Electron Sources for Ion Propulsion, II: Discharge Hollow Cathode, Journal of Propulsion and Power, 24(2008), pp. 866-879.

26) Katz, Ira., and Mikellides, I. G.: Neutral Gas Free Molecular Flow Algorithm Including Ionization and Walls for Use in Plasma Simulations, Journal of Computational Physics, 230(2011), pp. 1454-1464.

27) Mikellides, I. G.: Effects of Viscosity in a Partially-ionized Channel Flow with Thermionic Emission, Physics of Plasmas, 16(2009), pp. 013501.
28) Parra, F. I., Ahedo, E., Fife, J. M., and Martínez-Sánchez, M.: A Two-dimensional Hybrid Model of the Hall Thruster Discharge, Journal of Applied Physics, 100(2006), 023304.

29) Nanbu, K., Kitatani, Y.: An Ion-neutral Species Collision Model for Particle Simulation of Glow Discharge, Journal of Physics D, 28(1995), pp.324-330.

30) Bychenkov, V. Y., Silin, V. P., and Uryupin, S. A.: Ion-acoustic Turbulence and Anomalous Transport, Physics Reports, 164 (1988), pp. 119-215.

31) Goebel, D. M., and Katz, I.: Fundamentals of Electric Propulsion: Ion and Hall Thrusters, John Wiley \& Sons, Inc., New Jersey, 2008.

32) Amestoy, P. R., Duff, I. S., Koster, J., and L'Excellent, J. Y.: A Fully Asynchronous Multifrontal Solver Using Distributed Dynamic Scheduling, Journal of Matrix Analysis and Applications, 23(2001), pp. 15-41.

33) Goebel, D. M., Watkins, R. M., and Jameson, K. K.: LaB 6 Hollow Cathodes for Ion and Hall Thrusters, Journal of Propulsion and Power, 23(2007), pp. 552-558.

34) Goebel, D. M., and Chu, E.: High Current Lanthanum Hexaboride Hollow Cathodes for High Power Hall Thrusters, IEPC Paper 2011-053, 2011.

35) Jameson, K. K., Goebel, D. M., Mikellides, I., and Watkins, R. M.: Local Neutral Density and Plasma Parameter Measurements in a Hollow Cathode Plume, AIAA Paper 2006-4490, 2006.

36) Prewett, P. D., and Allen, J. E.: The Double Sheath Associated with a Hot Cathode, Proceedings of Royal Society of London, 348(1976), pp. 435-446.

37) Goebel, D. M., and Watkins, R. M.: Compact Lanthanum Hexaboride Hollow Cathode, Review of Scientific Instruments, 81(2010), 083504. 